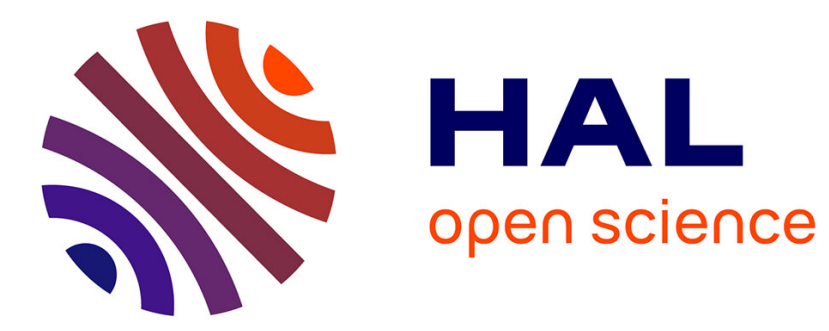

\title{
Les sécrétions digestives et leur régulation chez le jeune veau préruminant
}

\author{
P. Guilloteau, I. Le Huërou-Luron, J. Quillet, R. Toullec
}

\section{To cite this version:}

P. Guilloteau, I. Le Huërou-Luron, J. Quillet, R. Toullec. Les sécrétions digestives et leur régulation chez le jeune veau préruminant. Productions Animales, 1994, 7 (2), pp.85-95. hal-00896078

\section{HAL Id: hal-00896078 \\ https://hal.science/hal-00896078}

Submitted on 1 Jan 1994

HAL is a multi-disciplinary open access archive for the deposit and dissemination of scientific research documents, whether they are published or not. The documents may come from teaching and research institutions in France or abroad, or from public or private research centers.
L'archive ouverte pluridisciplinaire HAL, est destinée au dépôt et à la diffusion de documents scientifiques de niveau recherche, publiés ou non, émanant des établissements d'enseignement et de recherche français ou étrangers, des laboratoires publics ou privés. 
INRA Prod. Anim., $1994,7(2), 85$ - 95.
P. GUILLOTEAU,

I. LE HUËROU-LURON, J. QUILLET, R. TOULLEC

INRA Laboratoire du Jeune Ruminant 65 rue de St Brieuc 35042 Rennes Cedex
Le jeune veau reçoit un aliment liquide (lait ou lait de remplacement) qui passe directement dans la caillette grâce à la fermeture de la gouttière réticulaire. Cet état, dit préruminant, est maintenu jusqu'à l'abattage chez le veau de boucherie. La digestion des aliments se fait donc essentiellement sous l'action des sécrétions digestives de l'animal comme chez le monogastrique.

Au cours de son élevage, le veau doit s'adapter au système de production auquel il est soumis. En fonction de son âge, certaines périodes semblent plus délicates pour la production (phase périnatale, adaptation en atelier d'engraissement, maintien prolongé au stade préruminant, ...). Suite à la mise en place des quotas laitiers, rendant plus fluctuant la disponibilité et le prix de la poudre de lait écrémé, la nécessité d'introduire davantage de protéines de remplacement est redevenue un problème d'actualité. Ces protéines sont pourtant moins digestibles et peuvent provoquer des réactions d'intolérance, probablement de nature allergique. La moins bonne utilisation des aliments en fonction du stade de production et/ou de la nature des protéines alimentaires nécessite une meilleure connaissance de la physiologie digestive de l'animal et, en particulier, de l'action des sécrétions digestives et des mécanismes régissant leur synthèse et leur libération.

\section{Résumé}

Après la présentation de l'importance des sécrétions digestives pour transformer les aliments en nutriments absorbables et assimilables par l'organisme, les principes généraux de la régulation de leur synthèse et de leur libération sont décrits en soulignant la complexité des mécanismes. Des exemples sont donnés pour montrer l'effet de quelques facteurs (repas, nature des protéines, âge, digestibilité...) sur les sécrétions gastriques et pancréatiques et tenter de proposer une explication des mécanismes en cause. Quelques illustrations font état des apports possibles en nutrition et dans le domaine clinique chez l'animal et l'homme. En conclusion, les résultats récemment obtenus permettent, à l'aide des nouvelles techniques mises à notre disposition et associées à des méthodes classiques, d'ouvrir de nouvelles voies d'investigation dans le but d'intervenir sur la régulation des sécrétions pour mieux les adapter à l'aliment distribué, en tenant compte de l'environnement d'élevage.

\section{Les sécrétions digestives et leur régulation chez le jeune veau préruminant}

Dans cet article, après quelques rappels, nous présenterons des résultats récents obtenus chez le veau sur le rôle des sécrétions digestives, les principes généraux de la régulation de leur synthèse et de leur libération, l'effet de plusieurs facteurs en y incluant les mécanismes en cause (quelques exemples), l'utilisation de nouvelles méthodes d'investigation et leurs applications possibles et futures (quelques illustrations).

\section{1 / Rôle des sécrétions digestives}

Les sécrétions digestives jouent un rôle fondamental pour transformer les aliments en nutriments absorbables au niveau intestinal et directement assimilables par l'organisme. Lors de l'ingestion, les aliments subissent une légère insalivation, puis sont mélangés au suc gastrique dans la caillette. Après leur évacuation dans le duodénum, ils sont mélangés à la bile et au suc pancréatique déversés respectivement par le foie et le pancréas. Enfin, tout au long de lintestin, le suc intestinal y est rajouté. La production totale journalière des sécrétions digestives est estimée à $20-25 \mathrm{l} / 100 \mathrm{~kg}$ de poids vif chez le veau préruminant. Le premier effet de ces cinq sources de sécrétions digestives est, de par leur forte teneur en eau (tableau 1), de diluer les aliments pour faciliter le transit, l'homogénéisation du milieu et les dégradations. Celles-ci correspondent à une hydrolyse progressive des matériaux alimentaires suite à l'intervention des diverses enzymes contenues dans les sécrétions digestives. Mais ces enzymes ne peuvent être efficaces que si les caractéristiques du milieu, en particulier le $\mathrm{pH}$ des digesta, sont proches de l'optimum, ce qui constitue le troisième rôle des sécrétions digestives qui, par leur apport en électrolytes, modulent ces conditions. Enfin, les apports endogènes fournis par les sécrétions ne sont pas négligeables et sont réutilisés par l'animal en même temps que les apports alimentaires. On conçoit donc aisément que la composition chimique et biochimique de ces sécrétions détermine les étapes successives de la digestion. 
Tableau 1. Composition globale ( $\mathrm{g} / \mathrm{kg}$ de suc) du suc gastrique du veau préruminant recevant 2 repas par jour (Guilloteau 1986).

\begin{tabular}{|l|c|c|c|c|}
\hline $\begin{array}{l}\text { Origine de protéines } \\
\text { et teneurs dans l'aliment }\end{array}$ & Matière sèche & Azote & Lipides & $\begin{array}{c}\text { Somme des matières } \\
\text { minérales dosées }\end{array}$ \\
\hline - Poudre de lait écrémé & & & & \\
$15 \%$ de protéines & 10,39 & 0,40 & 0,17 & 7,36 \\
$25 \%$ de protéines & 9,51 & 0,37 & 0,13 & 7,21 \\
$28 \%$ de protéines & 10,73 & 0,35 & 0,13 & 7,17 \\
- Farine de poisson & 11,32 & 0,35 & 0,19 & 6,98 \\
$25 \%$ de protéines & 10,49 & 0,37 & 0,16 & 7,18 \\
Moyenne & & & & \\
\hline
\end{tabular}

Tableau 2. Caractéristiques des enzymes protéolytiques gastriques et pancréatiques.

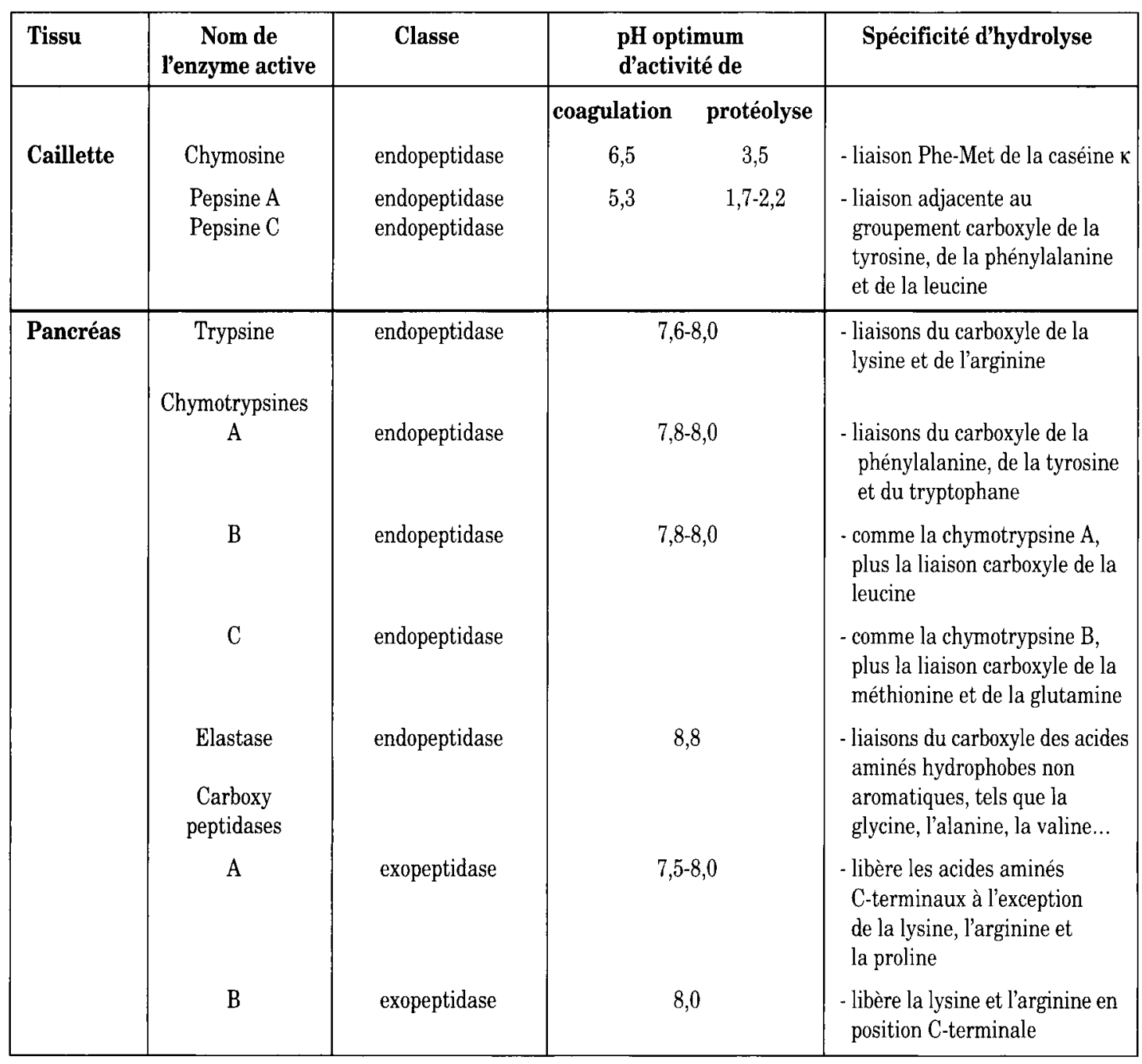

L'équipement enzymatique de la salive du veau est restreint puisqu'il se limite à la lipase pharyngiale (ou estérase prégastrique) et est dépourvu d'amylase (ou ptyaline). En revanche, les sucs gastrique et pancréatique sont très bien pourvus en enzymes digestives. Outre les enzymes protéolytiques (tableau 2), le suc gastrique contient les lysozymes $\mathrm{C}$ (agissant sur la paroi bactérienne mais en quantité peu importante chez le préruminant) et le suc pancréatique des enzymes lipolytiques (lipase et son cofacteur colipase, phospholipase A2 et cholestérol estérase), une enzyme glycolytique (amylase) et des enzymes nucléolytiques (ribonucléase et désoxyribonucléase). La bile ne contient pas d'enzymes. Les 
Figure 1. Principe général de la régulation des sécrétions digestives.

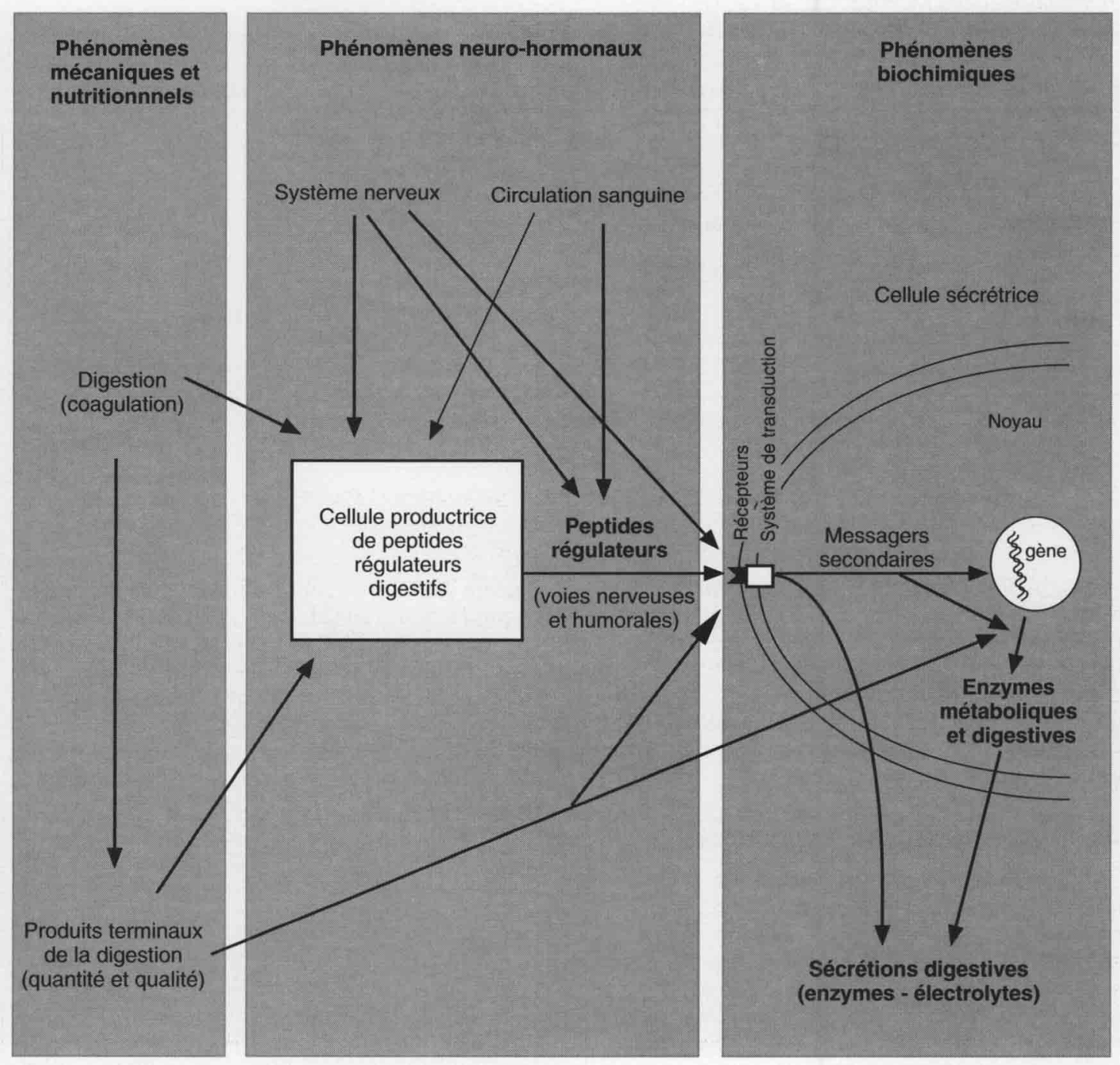

Tout stimulus est principalement transmis aux cellules digestives sécrétrices par des peptides régulateurs et/ou des neuropeptides.

enzymes intestinales sont essentiellement membranaires, à l'exception de l'iminodipeptidase (peptidase cytosolique). Des disaccharidases (lactase, maltase, isomaltase, thréhalase et cellobiase), des protéases (entérokinase, aminopeptidases A et $\mathrm{N}$, dipeptidyl-peptidase IV), une phosphomonoestérase (phosphatase alcaline) et des nucléases (5'-nucléotide phosphodiestérase et 5 ' nucléotidase) sont présentes dans la membrane de la bordure en brosse intestinale.

\section{2 / Régulation des sécrétions digestives : principes généraux}

Les sécrétions digestives sont sous la dépendance d'une régulation multifactorielle faisant intervenir des systèmes ou facteurs de différentes origines (mécanique, circulatoire, nerveux, hormonal...) et agissant simultanément (figure 1).

\section{1 / Aspects mécaniques et circulatoires}

Lorsque le jeune veau reçoit du lait entier ou un aliment dont les protéines sont apportées par de la poudre de lait, un coagulum ferme se forme au niveau de la caillette ; il se contracte rapidement en retenant les caséines et les lipides dont l'évacuation est lente et régulière. Lorsque la caséine est remplacée par des protéines de substitution non coagulables, tous les composants du digesta quittent la caillette sensiblement à la même vitesse et le temps de distension de cet organe est plus court (Toullec et Guilloteau 1989). La régulation mécanique de la digestion joue donc un rôle prédominant dans la régulation de la digestion (distension de la caillette, arrivée des digesta dans le duodénum, transit intestinal, absorption différentielle des nutriments...) et en particulier dans celle des sécrétions digestives.

Les modifications vasculaires influencent les phénomènes sécrétoires. A la suite d'un 
Tableau 3. Effets de quelques peptides régulateurs digestifs sur les sécrétions digestives chez les monogastriques (Thompson et al 1987).

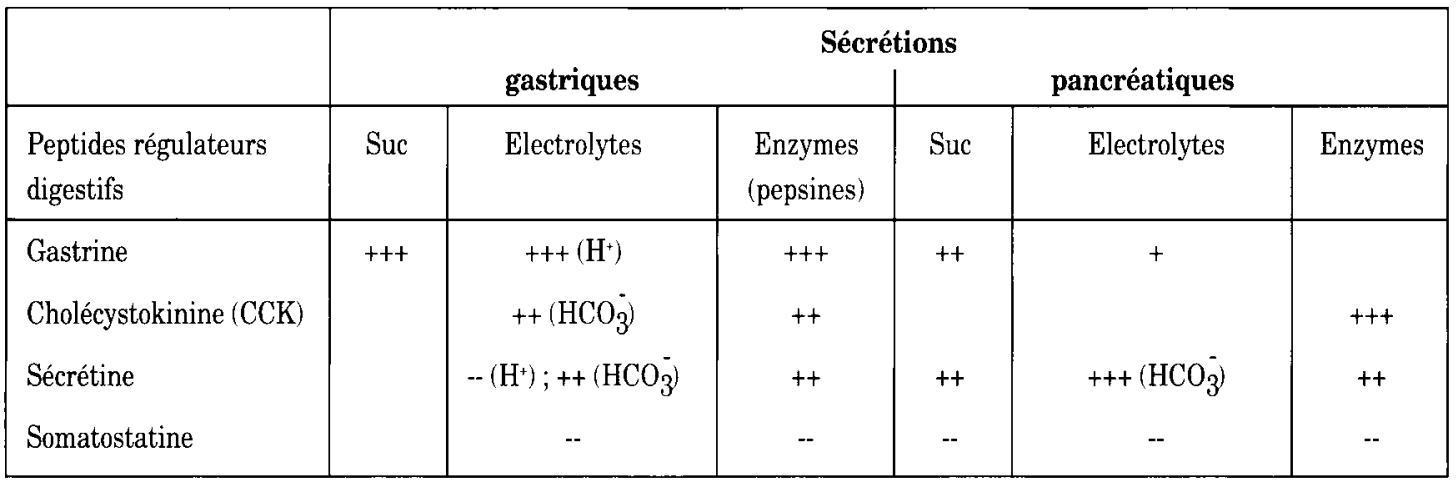

+ effet stimulateur; - effet inhibiteur. l'importance de l'effet augmente avec le nombre de signes.

Chaque peptide régulateur

intervient soit sur l'ensemble du tube digestif, soit plus particulièrement sur un organe, et interagit avec les autres peptides. repas, le débit sanguin augmente considérablement au niveau du tractus digestif. En revanche, en cas de jeûne, de stress, d'exercice important, etc, le sang peut être dirigé vers d'autres organes et ainsi diminuer l'irrigation du tractus digestif. En règle générale, le débit sanguin peut être accru par des agents humoraux et inhibé par voie nerveuse.

\section{2 / Aspects neuro-hormonaux}

Les cellules sécrétrices sont sous le contrôle du système nerveux central et du système nerveux périphérique (extrinsèque et intrinsèque). Chaque système est composé d'une structure parasympathique (centres moteur et sensitif vagaux, plexus myentériques et plexus sous-muqueux) et d'une structure sympathique (neurones médullaires sympathiques, plexus coeliaque). Les fibres parasympathiques vagales constituent les éléments de régulation les plus importants de l'organisme, en général, mais le système nerveux intrinsèque (plexus myentériques, etc) est essentiel à la fonction digestive et constitue un second niveau d'intégration des informations.

Les peptides régulateurs digestifs, dont le nombre d'acides aminés est généralement peu élevé ( $\leq 50-60)$, sont synthétisés par les cellules de différents organes (principalement celles du tube digestif). Leur rôle sur les sécrétions digestives a été étudié chez de nombreux mammifères (chat, chien, cobaye, hamster, homme, lapin, porc, rat, souris...) ; les effets physiologiques de quelques peptides sont rapportés dans le tableau 3 . Par exemple, la gastrine a un effet trophique sur l'ensemble du tube digestif, tandis que la cholécystokinine (CCK) stimule principalement le développement du pancréas et de la vésicule biliaire. Il est important de noter que de nombreuses interactions existent entre les peptides régulateurs digestifs. La plupart des effets physiologiques observés chez les monogastriques ont également été observés chez les ruminants. Ainsi, chez les veaux préruminants, l'infusion intraveineuse de CCK augmente la sécrétion d'acide gastrique, celles de sécrétine ou de glucagon la diminue (McLeay et al 1981). De plus, la gastrine, la CCK, la sécrétine et le peptide intestinal vasoactif (VIP) stimulent la sécrétion pancréatique, tandis que la somatostatine, le glucagon et le polypeptide pancréatique (PP) l'inhibent (Davicco et al 1979 et 1980, Pierzynowski et al 1991). Certains stimuli responsables de la sécrétion des peptides ont également été décrits chez le veau. L'acidification des contenus de la caillette et du duodénum entraîne une augmentation de la sécrétion de somatostatine.

Les hypothèses actuelles concernant la régulation digestive sont dominées par un concept global ne dissociant pas les aspects nerveux des aspects hormonaux (régulation neuro-hormonale) et faisant également intervenir le système immunitaire (par exemple l'histamine, qui est sécrétée par les mastocytes, agit sur la sécrétion acide de la caillette).

\section{3 / Aspects biochimiques cellulaires}

Les peptides régulateurs digestifs transportés par voie humorale et/ou nerveuse, transmettent le signal à la cellule productrice de sécrétions digestives par l'intermédiaire de leurs récepteurs membranaires. Le message recueilli est alors véhiculé par les messagers secondaires membranaires et intracellulaires. Cette cascade de réactions biochimiques se traduit par une régulation à différents niveaux de la synthèse protéique (figure 1).

Tout stimulus appliqué au niveau périphérique (repas, nature des protéines alimentaires,...) produit des messages qui sont transmis aux cellules digestives sécrétrices d'eau, de mucus, d'enzymes et/ou d'électrolytes, par les peptides régulateurs digestifs et/ou les neuropeptides (figure 1 ). L'étape de régulation peut se situer au niveau de la synthèse des peptides régulateurs responsables du message, au niveau des récepteurs de la transduction et des messagers secondaires, au niveau du gène 
Figure 2. Evolution postprandiale des concentrations plasmatiques de quelques peptides régulateurs digestifs et des sécrétions gastriques et pancréatiques chez le veau préruminant (Ternouth et Buttle 1973, Guilloteau 1986).

Gastrine (pg/ml)

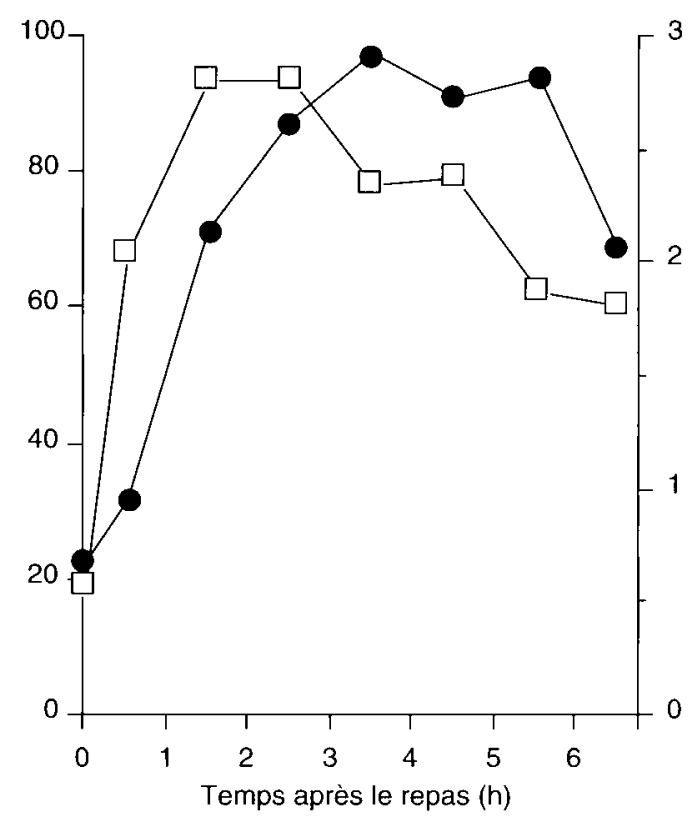

$\%$ de la valeur prépandiale

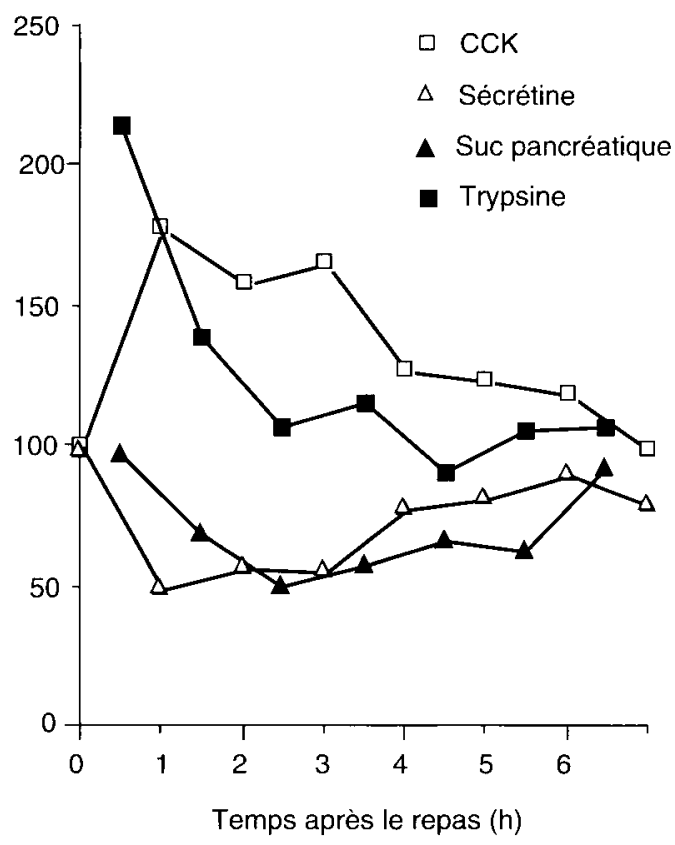

ou des ARNm spécifiques des enzymes pancréatiques (synthèse protéique) et/ou à une étape postérieure sur les mécanismes sécrétoires ; elle aboutit à une modification des sécrétions digestives (quantité et qualité) dépendant des stimuli.

\section{3 / Régulation des sécrétions digestives : exemples}

De par l'ensemble des mécanismes mis en jeu, la régulation des sécrétions digestives est diversifiée et complexe ; nos connaissances dans ce domaine sont également très incomplètes. Pour simplifier, nous nous limiterons à quelques exemples en nous appuyant sur des résultats obtenus chez le veau et parfois en faisant appel à des concepts généraux proposés chez les mammifêres monogastriques. L'illustration portera le plus souvent sur les variations de sécrétions gastriques et pancréatiques associées à des variations parallèles de taux plasmatiques de plusieurs peptides. Une certaine prudence doit nuancer les hypothèses émises si elles ne sont supportées que par cette approche. Toutefois, quelques résultats mettant en évidence l'effet direct de peptides régulateurs sur la sécrétion gastrique ou pancréatique rapporté dans la deuxième partie pour quelques-uns, confortent les hypothèses présentées ci après.

\section{1 / Sécrétions digestives et repas}

L'augmentation du volume du suc gastrique sécrété et de son contenu (électrolytes dont $\mathrm{HCl}$, chymosine et pepsine) au cours des 2 à $3 \mathrm{~h}$ qui suivent le repas (Guilloteau et Toullec 1983) est à relier avec l'accroissement postprandial du taux de gastrine (Bloom et al 1978, Toullec et al 1992) (figure 2).

En revanche, la réponse de la sécrétion pancréatique en fonction du repas varie selon les techniques expérimentales utilisées. Selon Pierzynowski et al (1991), chez des veaux âgés de 6 semaines, une augmentation du flux de suc se produit pendant le repas et n'existerait plus 30 min après; selon d'autres auteurs (Ternouth et Buttle 1973, Ternouth et al 1975, Khorasani et al 1989), l'effet du repas aurait lieu pendant les 6 premières heures postprandiales chez des animaux âgés de moins d'un mois. Ces données pourraient s'accorder avec celles de Davicco (1978) qui, chez des veaux de même âge, montrent que la stimulation pancréatique (suc, minéraux, protéines et activité amylasique) se produirait en deux pics : le premier 15 à 30 min et le second 3 à $6 \mathrm{~h}$ après le repas ; en revanche, nos premiers résultats semblent montrer une diminution importante du volume de suc pendant la première heure postprandiale et un retour au niveau basal 4 à $5 \mathrm{~h}$ après le repas (Le Huërou-Luron et al 1994). La concentration de protéines du suc serait augmentée (Gorrill et al 1967, Pierzynowski et al 1991) mais pas celle de trypsine (Pierzynowski et al 1991). L'influence du repas sur la sécrétion pancréatique serait probablement liée à une stimulation du nerf vague qui potentialiserait l'effet de la CCK et surtout celui de la sécrétine (Zabielski et al 1990, Pierzynowski et al 1991) (figure 2). Toutefois nos connaissances concernant l'effet du repas sur les sécrétions pancréatiques restent très limitées ; elles doivent être améliorées par des études complémentaires incluant des protocoles permettant de mieux comprendre les mécanismes de régulation.

\section{Si l'effet du repas sur la sécrétion du suc gastrique est très net, l'effet sur la sécrétion pancréatique est moins facile ̀̀ interpréter.}




\section{2 / Sécrétions digestives et nature des protéines}

Lorsque le veau ingère des protéines de substitution, celles-ci ne coagulent pas dans la caillette. Elles sont en conséquence évacuées plus rapidement et moins hydrolysées (Guilloteau et al 1975). Cette régulation mécanique entraîne un afflux beaucoup plus important des composants intacts de l'aliment dans le duodénum, qui peut ainsi modifier l'équilibre neuro-endocrinien du veau.

Les quelques résultats disponibles concernent les protéines de poisson et surtout celles de soja. Avec les premières, il ne semble y avoir aucune modification de la sécrétion abomasale (suc, composition, quantité d'enzymes et d'électrolytes) chez des veaux porteurs d'une poche gastrique (Guilloteau 1986). De même, les modifications observées au niveau du suc pancréatique sécrété au cours des $6 \mathrm{~h}$ postprandiales semblent mineures (augmentation seulement des quantités de lipase et de RNAse) (Ternouth et al 1975). Cependant, la mesure des taux circulants des peptides régulateurs digestifs montre que les protéines de poisson entraînent une augmentation des concentrations plasmatiques de CCK et de VIP, mais une diminution de celles de somatostatine et surtout de sécrétine (Guilloteau et al 1984, Guilloteau 1986).

Chez le veau préruminant, la réponse pancréatique à l'ingestion de régimes contenant des protéines de soja ayant subi des traitements technologiques différents est controversée. Gorrill et al (1967), Ternouth et al (1974), Guilloteau et al (1986) et Khorasani et al (1989) rapportent une diminution des sécrétions pancréatiques et/ou des activités enzymatiques tissulaires chez des veaux ayant reçu des produits issus du soja. En revanche, Kakade et al (1976) ne trouvent pas de modification pour les concentrations des protéines dans le suc et les activités enzymatiques du tissu. Ces résultats non concordants pourraient s'expliquer par l'utilisation de méthodologies expérimentales différentes, mais aussi par la nature des produits issus du soja et les traitements technologiques qui leur sont appliqués. Ainsi, la composition en facteurs antinutritionnels varie et, par exemple, les activités antitrypsiques peuvent être 9 fois plus faibles dans un isolat de soja chauffé que dans un tourteau délipidé. Il semble toutefois difficile de reproduire avec fiabilité des traitements technologiques. Par exemple, Le Dréan et al (1994) n'obtiennent pas de variations du poids du pancréas et de son contenu enzymatique aussi marquées que Guilloteau et al (1986) avec un aliment contenant des protéines de soja préparées de façon similaire. Néanmoins, la plupart des données bibliographiques montrent que les protéines de soja ont une action négative sur la sécrétion pancréatique par rapport à celles du lait. Les relations entre la réponse pancréatique et le taux circulant de peptides régulateurs digestifs ne sont pas évidentes (figure 2). En effet, le poids du pancréas est plus faible chez les animaux nourris avec du soja que chez ceux recevant des pro- téines de lait, bien que leurs teneurs plasmatiques en peptides régulateurs digestifs ayant un effet trophique sur le pancréas soient identiques (gastrine) ou plus élevées (CCK). De même, si l'effet négatif sur le contenu enzymatique du pancréas est en accord avec la diminution de sécrétine, il ne l'est pas avec l'augmentation de la concentration de CCK (Guilloteau et al 1986). Il semble donc qu'il ne soit pas possible d'expliquer tous les phénomènes observés uniquement par les variations des taux de peptides régulateurs, sans envisager les différentes étapes de régulation dans lesquelles ils sont impliqués. Ainsi, une étude récente ayant pour objectif d'approfondir les mécanismes mis en cause, montre que les niveaux d'ARNm spécifiques de l'amylase, de la trypsine et de la chymotrypsine pancréatiques tendent à être plus élevés avec des protéines de soja qu'avec celles du lait (Le Dréan et al 1994) ; ceci pourrait être lié aux valeurs postprandiales plus élevées de la CCK. La régulation s'effectuerait donc à un autre niveau et/ou par l'intervention d'autres mécanismes.

\section{3 / Sécrétions digestives, âge et digestibilité}

L'infusion duodénale d'aliments contenant des protéines de lait ou des protéines de poisson à une vitesse similaire à celle observée lors des études d'évacuation gastrique entraîne une diminution de la digestibilité des matières azotées (de $4 \%$ ), des matières grasses (de 11 à $14 \%$ ) et des minéraux (de 9 à $15 \%$ ) (Guilloteau et al 1981). La non-réintroduction du suc pancréatique pendant $12 \mathrm{~h}$ au cours de la journée provoque également une baisse de digestibilité des matières azotées ( $8 \%$ ) et des lipides $(6 \%)$ (Ternouth et al 1974). Les sécrétions gastriques et pancréatiques ont donc un rôle important dans la digestion globale des constituants du régime.

La digestibilité des protéines du lait et du lactosérum évolue au cours du premier mois de vie (de 0,86 à 0,94 d'après Grongnet et al 1981). Pendant la même période, le flux total de suc pancréatique mesuré avant le repas semble augmenter (Gorrill et al 1967). Il en est de même pour le contenu de la plupart des enzymes pancréatiques mesuré à jeun à partir du $2^{\text {ème }}$ jour de vie (trypsine, chymotrypsine, élastase, carboxypeptidases A et B, ribonucléase, $\alpha$ amylase, lipase...). Par ailleurs, à la naissance (P. Guilloteau et al résultats non publiés), les concentrations plasmatiques de l'ensemble des peptides régulateurs digestifs sont fortement modifiées, ce qui pourrait être lié à un bouleversement des équilibres hormonaux en relation avec le stress provoqué par le passage d'un environnement protégé à la vie aérienne. Au cours des trois premières semaines qui suivent la naissance, des changements importants sont observés dans l'évolution des taux plasmatiques basaux des peptides dosés, à l'exception du VIP. A l'âge de $21 \mathrm{j}$, les taux de sécrétine, de CCK et de PP sont plus élevés qu'à la naissance, tandis que ceux de gastrine, de motiline et de somatostatine 
Figure 3. Evolution des concentrations plasmatiques de quelques peptides régulateurs digestifs et quelques activités enzymatiques gastriques et pancréatiques (en \% des valeurs à 2j) chez le veau préruminant (Guilloteau et al 1992a et 1992b, Le Huërou-Luron et al 1992, Toullec et al 1992). * significatif à $P<0,05$ entre les valeurs à 1 ou $2 j$ et celles à $21,28,91$ et $119 j$.

Concentration plasmatique $(\mathrm{pg} / \mathrm{ml})$

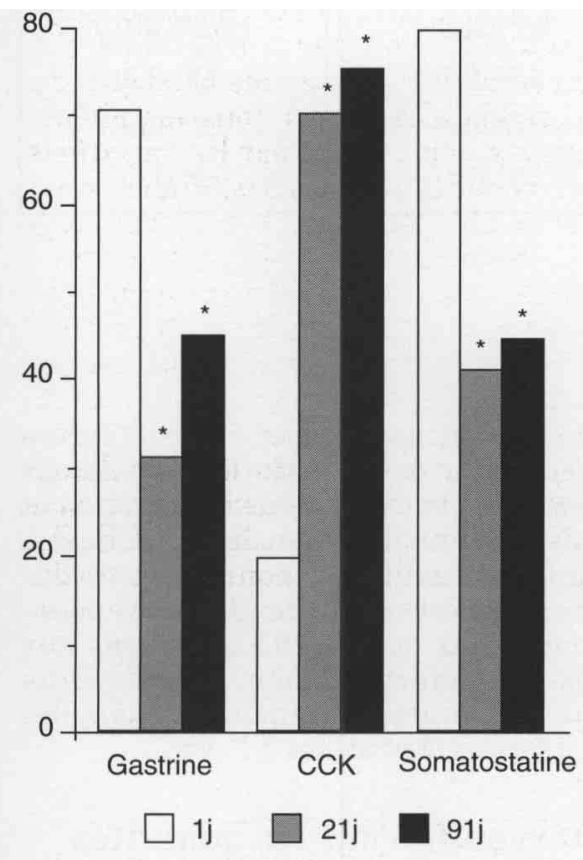

Activité enzymatique / kg PV

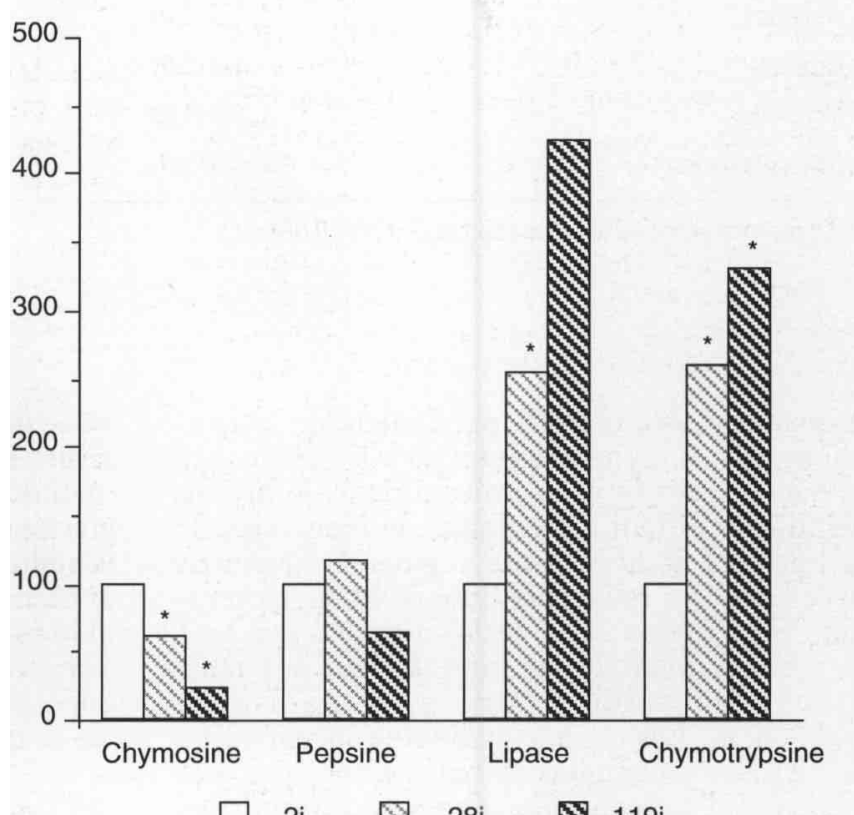

2j
$28 j$ 119j sont plus faibles. La diminution est particulièrement rapide pour la gastrine et la somatostatine puisqu'elle a lieu surtout au cours des 5 premiers jours de vie (Guilloteau et al 1992a, Toullec et al 1992). Au delà de l'âge de trois semaines, les teneurs plasmatiques en peptides régulateurs digestifs semblent se stabiliser.

Les taux élevés de CCK et de sécrétine en fin de la période embryonnaire peuvent expliquer en partie le stock d'enzymes accumulées dans le pancréas à la naissance ; en revanche, les taux élevés de CCK, sécrétine et VIP observés après l'ingestion des premiers repas de colostrum (Guilloteau et al 1992b) peuvent être la cause de la diminution du contenu enzymatique du pancréas au cours des 24 premières heures de vie chez le jeune ruminant (Guilloteau et al 1984, Le Huërou-Luron et al 1992), en favorisant la libération des enzymes stockées en fin de période foetale. Chez le jeune veau, la diminution rapide du taux de gastrine après la naissance favoriserait celle de chymosine dans la caillette et la stagnation des sécrétions de pepsine et d'HCl (Guilloteau et al 1980). De même, l'accroissement de la taille du pancréas et l'augmentation des activités enzymatiques (Ternouth et Buttle 1973, Guilloteau et al 1984, Le Huërou-Luron et al 1992) sont parallèles à l'évolution du taux de CCK et de sécrétine, celui de somatostatine diminuant simultanément (figure 3 ). En revanche, les faibles modifications observées pour la plupart des activités enzymatiques pancréatiques chez les veaux préruminants entre les âges de 4 et 17 semaines pourraient être en relation avec le taux élevé de somatostatine plasmatique qui inhiberait l'effet stimulateur de la CCK.
Cependant, l'effet physiologique des peptides régulateurs digestifs dépend fortement de la présence et de la fonctionnalité de récepteurs spécifiques dans les glandes et tissus digestifs. Des récepteurs pancréatiques VIPergiques fonctionnellement couplés à l'adénylate cyclase ont été mis en évidence à la naissance et aux âges de 4 et 17 semaines. Toutefois, à la naissance, il n'existe qu'une seule classe de sites de liaison de forte affinité et de basse capacité pour le VIP, mais une deuxième classe de sites de liaison de faible affinité et de haute capacité est observée à partir de l'âge de 4 semaines (Le Meuth et al 1991). Les récepteurs pancréatiques CCK/gastrine sont fonctionnellement couplés aux protéines $\mathrm{G}$; leur existence sous forme de deux classes de sites de liaison (une de forte affinité et de basse capacité et une autre de faible affinité et de haute capacité pour la CCK-9) a été démontrée dès la naissance (Le Meuth et al 1993). Par ailleurs, pour la première fois, une expression différentielle de deux sous-types de récepteurs CCK/gastrine de masses moléculaires différentes a été mise en évidence au cours de l'ontogénèse. Chez les veaux nouveaunés, les récepteurs $\mathrm{CCK} /$ gastrine pancréatiques appartiennent principalement au soustype CCK-A (liant la CCK avec une forte affinité et la gastrine avec une faible affinité) de $85-95 \mathrm{kDa}$, tandis que chez les veaux âgés de 4 et 17 semaines, les récepteurs $\mathrm{CCK} /$ gastrine du sous-type CCK-B (liant la CCK et la gastrine avec des affinités voisines) de $42-47 \mathrm{kDa}$, sont prédominants.

Enfin, le message transmis par les récepteurs aux peptides régulateurs digestifs via les

\section{Les teneurs plasmatiques en peptides régulateurs évoluent beaucoup au cours des 3 semaines après la naissance, puis se stabilisent.}


Tableau 4. Variations du niveau relatif des ARN messagers et des activités spécifiques de quatre enzymes pancréatiques au cours du développement postnatal chez le veau préruminant (Le Huërou et al 1990).

\begin{tabular}{|l|c|c|c|}
\hline \multirow{2}{*}{$\begin{array}{l}\text { Niveau relatif des } \mathbf{A R N}_{\mathbf{m}} \text { spécifiques } \\
\text { (et des activités enzymatiques) }\end{array}$} & $\mathbf{0}$ & $\mathbf{2 8}$ & Age (j) \\
\cline { 2 - 4 } & 119 \\
\hline Amylase & $1,0(1,0)$ & $2,7^{*}(23,0)$ & $10,2^{*}\left(42,0^{*}\right)$ \\
Lipase & $1,0(1,0)$ & $2,5^{*}(0,8)$ & $8,3^{*}(1,3)$ \\
Trypsine & $1,0(1,0)$ & $3,0^{*}(0,9)$ & $4,9^{*}\left(0,4^{*}\right)$ \\
Chymotrypsine & $1,0(1,0)$ & $0,6^{*}(1,3)$ & $0,9^{*}\left(1,5^{*}\right)$ \\
\hline
\end{tabular}

* Différence significative par rapport à $0 \mathrm{j}(\mathrm{P} \leq 0,05)$.

messagers secondaires, peut moduler la synthèse des enzymes digestives à différents niveaux. Chez le veau préruminant, le niveau relatif des ARNm spécifiques de l'amylase, de la lipase et de la trypsine augmente fortement avec l'âge ; en revanche, celui de la chymotrypsine (Le Huërou et al 1990) diminue pendant le premier mois de vie puis devient à 4 mois proche de celui trouvé à la naissance (tableau 4). Les changements de concentration des ARNm spécifiques de ces enzymes peuvent résulter d'un contrôle transcriptionnel des gènes correspondants et/ou d'une modulation de la maturation, du transport ou de la stabilité des messagers. Ce contrôle prétraductionnel s'accompagne d'une régulation traductionnelle. En effet, des variations non parallèles, voire opposées, du niveau des ARNm et des activités sont observées. Ainsi, l'activité de la trypsine $(x 0,4)$ et la quantité de son ARNm (x 5) varient en sens inverse durant toute la période postnatale.

En résumé, la digestibilité des constituants de l'aliment semble être liée, au moins en partie, à la composition en enzymes digestives des sécrétions pancréatiques. La digestibilité, le flux pancréatique et le contenu en enzymes du tissu augmentent simultanément surtout au cours du premier mois de vie. Parallèlement, il est observé, pendant cette période, une évolution des taux plasmatiques de peptides régulateurs et de leurs récepteurs, ainsi qu'une modification des quantités d'ARNm de quatre enzymes pancréatiques. L'hypothèse d'une régulation par les peptides régulateurs digestifs et/ou via d'autres voies, sur la synthèse et la sécrétion enzymatiques peut donc être envisagée. Des études complémentaires sont nécessaires pour en expliquer les mécanismes.

\section{4 / Perspectives d'applications utilisant les nouvelles méthodes d'investigation}

Les nouvelles méthodes d'investigation permettent d'étudier les mécanismes qui régulent les sécrétions enzymatiques aux niveaux cellulaire et moléculaire (voir exemples rapportés dans la partie précédente), contribuant à augmenter nos connaissances sur la cascade de réactions qui sont impliquées. Il reste encore difficile d'en tirer des applications pratiques immédiates dans le domaine de la nutrition et de l'alimentation du veau de boucherie. Cependant, une meilleure connaissance des mécanismes devrait ouvrir de nouvelles possibilités pour mieux adapter les sécrétions aux conditions de production. Pour illustrer cette idée, nous allons prendre quelques exemples de ce qui pourrait être utilisé à l'avenir.

\section{1 / Intervention sur les quantités d'enzymes sécrétées}

L'introduction de protéines de soja semble diminuer la sécrétion pancréatique, entraînant une moins bonne valorisation de l'aliment. Les résultats récemment acquis (qui ne concernent qu'une partie des mécanismes mis en jeu) peuvent laisser entrevoir une utilisation de peptides régulateurs digestifs ou de leurs agonistes pour stimuler la sécrétion pancréatique. Par exemple, chez le rat, une injection de céruléine, un analogue de la CCK, induit une augmentation de la biosynthèse des enzymes protéolytiques et des injections répétées induisent une hypertrophie et une hyperplasie pancréatique (Barrowman et Mayston 1974). Pour la famille CCK/gastrine, il semblerait que seuls des peptides à effet CCK puissent agir chez le veau nouveau-né (présence de récepteurs uniquement de type CCK-A) alors que des peptides à effet CCK et/ou gastrine pourraient être utilisés chez des veaux plus âgés (présence de récepteurs de types CCK-A et $\mathrm{B}$ ).

Cette technique aurait l'inconvénient de rappeler l'emploi des anabolisants et les contraintes de production résultantes. Ces aspects négatifs pourraient être surmontés en favorisant une production adéquate de peptides régulateurs digestifs endogènes par l'introduction de «molécules naturelles" dans l'alimentation du veau. Par exemple, il vient d'être démontré que la présence de produits issus de la digestion gastrique des caséines dans le duodénum proximal, stimule fortement la production de CCK endogène chez le rat (les taux plasmatiques sont multipliés par 3) (Beucher 1993). Des études ont été et seront menées dans ce sens chez le veau, tout en vérifiant l'effet global sur la production de viande de qualité. 


\section{2 / Intervention sur la nature des enzymes sécrétées}

La plupart des protéines de réserve, dont celles de la graine de soja, sont de type globulaire. Elles sont beaucoup moins digestibles que les caséines du fait de leur structure. Leur hydrolyse par les enzymes du suc pancréatique est insuffisante et des fragments peuvent entraîner des troubles de nature allergique, même après avoir subi des traitements technologiques. L'hypothèse d'une meilleure dénaturation et hydrolyse par un suc digestif dont la composition serait mieux adaptée, serait intéressante. Par exemple, si l'augmentation de la teneur du suc pancréatique en élastase II, qui agit spécifiquement sur les liaisons hydrophobes des protéines globulaires, aboutit à une meilleure hydrolyse des protéines, une connaissance des mécanismes de sa synthèse et de sa libération permettrait d'intervenir pour en favoriser la production. L'hydrolyse plus complète des protéines, en particulier celles à caractère allergénique, pourrait limiter et/ou annuler leurs interactions négatives avec le tube digestif. Une action au niveau de la régulation globale (peptides régulateurs digestifs, substance d'origine nutritionnelle...) et moléculaire (régulation au niveau des gènes, production d'animaux transgéniques,...) pourrait être ainsi envisagée en évitant les traitements technologiques élaborés qui coûtent cher et qui entraînent parfois des modifications dans la disponibilité des nutriments pour l'animal.

Dans un avenir plus lointain, même si cela peut paraître futuriste, on peut envisager la production de veaux transgéniques, porteurs d'enzymes spécifiques pour le ou les substrats à faire ingérer. Ainsi une expérience réalisée en Angleterre vient de montrer qu'il était possible d'obtenir des souris productrices d'endoglucanase $\mathrm{E}$ pancréatique rendant l'animal capable d'utiliser la cellulose ; les auteurs parlent ainsi de souris "ruminantes» (Hall et al 1993).

\section{3 / Utilisation à titre préventif ou curatif (en clinique vétérinaire et/ou humaine)}

Chez le veau âgé de moins d'un mois, une modification des sécrétions digestives pourrait être envisagée à titre préventif pour mieux valoriser l'aliment pendant les premiers mois de vie postnatale. Il pourrait en être de même lors de l'entrée en atelier d'engraissement qui entraîne toujours des troubles digestifs ; cette situation pourrait d'ailleurs être assimilée à des soins vétérinaires chez l'animal, en administrant par exemple de l'octréotide, un analogue de la somatostatine, qui a un effet bénéfique dans de nombreux types de diarrhées chez l'homme (Harris et Kokoris 1993).
La découverte récente chez le veau de récepteurs pancréatiques de type CCK-B, largement majoritaire par rapport au type CCK-A, fait de cet animal un modèle de choix, à application potentielle en clinique humaine, pour étudier leurs caractéristiques biochimiques et fonctionnelles puisque, en l'état actuel des connaissances, c'est le seul mammifêre avec l'homme qui présente cette particularité (Lee et al 1993).

\section{4 / Ouverture de nouvelles voies de recherches}

La cascade des événements régissant les phénomènes de régulation est loin d'être connue dans sa totalité, ce qui ne permet pas d'intervenir encore chez l'animal ou l'homme de façon rapide et efficace. Les résultats récents permettent de suggérer de nouvelles études qui apporteraient des maillons indispensables, c'est-à-dire une meilleure connaissance des mécanismes de transduction et les messagers secondaires présents dans les cellules sécrétrices. D'ailleurs, de nouveaux outils méthodologiques sont maintenant à notre disposition (clonage des récepteurs CCK $\mathrm{A}$ et $\mathrm{B}$, cellules transfectées, culture de cellules eucaryotes ayant incorporé de façon permanente les ADNc correspondant aux récepteurs..), sans oublier les techniques plus classiques (acini isolés, animal entier cathétérisé...). Tout cela doit encourager une recherche cognitive en même temps qu'une recherche appliquée puisqu'elles ont des intérêts communs et complémentaires.

\section{Conclusion}

Dans les phénomènes de la digestion, les sécrétions digestives se révèlent être d'une importance capitale chez le jeune veau préruminant. Rappelons simplement que le volume total de ces sécrétions représente 2 à 3 fois les quantités d'eau ingérées par jour. Il est nécessaire de connaître les mécanismes qui régissent ces sécrétions, afin de pouvoir agir en vue d'une meilleure adaptation de l'organisme aux aliments qui lui sont fournis. Grâce à la modification de paramètres liés à l'aliment et/ou à l'animal, cette adaptation doit faire intervenir au maximum les facteurs endogènes pour respecter le plus possible les mécanismes physiologiques. Les applications en terme de rentabilité économique immédiate ou à court terme ne sont pas toujours évidentes, mais sousjacentes; les résultats offrent cependant des perspectives intéressantes dans le domaine de la nutrition animale et humaine, ainsi que dans celui de la santé. 


\section{Références bibliographiques}

Barrowman J.A., Mayston P.D., 1974. The trophic influence of cholecystokinin on the rat pancreas. $\mathrm{J}$ Physiol. (London) 238, 73P-75P.

Beucher S., 1993. Effet des produits de la digestion gastrique des caséines sur les sécrétions biliopancréatiques chez le porc et sur la libération de cholécystokinine (CCK) par les cellules intestinales de rat. Thèse de doctorat, Université Paris VII, $133 p$.

Bloom S.R., Edwards A.V., Hardy R.N., 1978. The role of autonomic nervous system in the control of pancreatic endocrine responses to milk ingestion in the calf. J. Physiol. (London), 280, 37-53.

Davicco M.J., 1978. Régulation du pancréas exocrine $\mathrm{du}$ jeune veau. Thèse de doctorat, Université Clermont II, $\mathrm{n}^{\circ} 542,102 \mathrm{p}$.

Davicco M.J., Lefaivre J., Thivend P., Barlet J.P., 1979. Exocrine pancreatic secretion in preruminant milk-fed calves. Ann. Rech. Vét. 10, 428-430.

Davicco M.J., Lefaivre J., Barlet J.P., 1980. The endocrine regulation of exocrine pancreas in preruminant milk-fed calves. Ann. Rech. Vét. 11, $123-132$

Gorrill A.D.L., Thomas J.W., Stewart W.E., Morrill J.L., 1967. Exocrine pancreatic secretion by calves fed soybean and milk protein diets. J. Nutr. 92, 86-92.

Grongnet J.F., Patureau-mirand P., Toullec R. Prugnaud J., 1981. Utilisation des protéines du lait et du lactosérum par le jeune veau préruminant. Influence de l'âge et de la dénaturation des protéines du lactosérum. Ann. Zootech. 30, 443-464.

Guilloteau P., 1986. Digestion des protéines chez le jeune ruminant. Thèse de Doctorat d'Etat, Université Pierre et Marie Curie, Paris VI, 242p.

Guilloteau P., Toullec R., 1983. Circadian changes in the abomasal secretions of the preruminant calf. Reprod. Nutr. Dévelop. 23, 967-977.

Guilloteau P., Paruelle J.L., Toullec R., Mathieu C.M., 1975. Utilisation des protéines par le veau préruminant à l'engrais. III. Influence du remplacement des protéines du lait par celles du poisson sur la vidange stomacale. Ann. Zootech. 24, 243-253.

Guilloteau P., Toullec R., Garnot P., Martin P. Brulé G., 1980. Influence de l'âge sur les sécrétions de suc gastrique et d'électrolytes chez le veau préruminant. Reprod. Nutr. Dévelop. 20, 1279-1284.

Guilloteau P, Toullec R., Patureau-Mirand P., Prugnaud J., 1981. Importance of the abomasum in the digestion in preruminant calf. Reprod. Nutr. Dévelop. 21, 885-899.

Guilloteau P., Corring T., Toullec R., Robelin J., 1984. Enzyme potentialities of the abomasum and pancreas of the calf. I. Effect of age in the preruminant. Reprod. Nutr. Dévelop. 24, 315-325.

Guilloteau P., Corring T., Chayvialle J.A., Bernard C., Sissons J.W., Toullec R., 1986. Effect of soya protein on digestive enzymes, gut hormone and anti-soya antibody plasma levels in the preruminant calf Reprod. Nutr. Develop. 26, 717-728.
Guilloteau P., Le Huërou-Luron I., Chayvialle J.A., Mouats A., Bernard C., Cuber J.C., Burton J., Puigserver A., Toullec R., 1992a. Plasma and tissue levels of digestive regulatory peptides during postnatal development and weaning in the calf. Reprod. Nutr. Develop. 32, 285-296.

Guilloteau P., Le Huërou-Luron I., Toullec R. $1992 \mathrm{~b}$. Patterns of gut regulatory peptides and of some receptors in calves: effects of age, weaning and feeding. In: Nutrition-related endocrine changes in cattle (M. Stangassinger \& J.W. Blum, eds), University of Berne, 44-53.

Hall J., Ali S., Surami M.A., Hazlewood G.P., Clark A.J., Simons J.P., Hirst B.H., Gilbert H.J., 1993. Manipulation of the repertoire of digestive enzymes secreted into the gastrointestinal tract of transgenic mice. Biotechnology 11, 376-379.

Harris A.G., Kokoris S.P., 1993. Application thérapeutique de la somastostatine et de son analogue, l'octréotide. La Presse Médicale, 22, 724. 733.

Kakade M.L., Thompson R.D., Eengelstad W.E., Behrens G.C., Yoder R.D., Crane F.M., 1976. Failure of soyabean trypsin inhibitor to exert deleterious effects in calves. J. Dairy Sci. 59, 1484-1489.

Khorasani G.R., Ozimek L., Sauer C., Kennelly J.J., 1989. Substitution of milk protein with isolated soy protein in calf milk replacers. J. Anim. Sci. 67, 16341641.

Le Dréan G., Le Huërou-Luron I., Philouze-Rome V., Toullec R., Guilloteau P., 1994. Pancreatic nutritional adaptation in preruminant calves: effects of different soyabean diets. Ann. Zootech., sous presse.

Lee Y.M., Beinborn M., Mc Bride E., Lu M., Kolakowski L.F., Kopin A.S., 1993. The human brain cholecystokinin-B/gastrin receptor. Cloning and characterization. J. Biol. Chem. 268, 8164-8169.

Le Huërou I., Wicker C., Guilloteau P., Toullec R., Puigserver A., 1990. Specific regulation of the gene expression of some pancreatic enzymes during postnatal development and weaning in the calf. Biochim. Biophys. Acta 1048, 257-264.

Le Huërou-Luron I., Guilloteau P. WickerPlanquart C., Chayvialle J.A., Burton J.H., Mouats A., Toullec R., Puigserver A., 1992. Gastric and pancreatic enzyme activities and their relationship with some gut regulatory peptides during postnatal development and weaning in calves. J. Nutr. 122 , 1434-1445.

Le Huërou-Luron I., Guilloteau P., Chayvialle J.A., Le Dréan G., Gestin M., Romé V., Toullec R., 1994. Effect of meal on pancreatic exocrine secretion and on secretin plasmatic level of preruminant calf. Preliminary results. Ann. Zootech., sous presse.

Le Meuth V., Farjaudon N., Bawab W., Chastre E., Rosselin G., Guilloteau P., Gespach C., 1991. Characterization of binding sites for VIP related peptides and activation of adenylate cyclase in developing pancreas. Am. J. Physiol. 260, G265G274. 
Le Meuth V., Philouze-Romé V., Le Huërou-Luron I., Formal M., Vaysse N., Gespach C., Guilloteau P., Fourmy D., 1993. Differential expression of A and B subtypes CCK/gastrin receptors in the developing calf pancreas. Endocrinology 133, 1182-1191.

Mc Leay L.M., Bell F.R., 1981. Effect of cholecystokinin, secretin, glucagon, and insulin on gastric emptying and acid secretion in the calf. Am. J. Vet. Res. 41, 1590-1594.

Pierzynowski S.G., Zabielski R., Weström B.R., Mikolajczyk M., Barej W., 1991. Development of the exocrine pancreatic function in chronically cannulated calves from the preweaning period up to early rumination. J. Anim. Physiol. Anim. Nutr. 65, 165-172.

Ternouth J.H., Buttle H.L., 1973. Concurrent studies on the flow of digesta in the duodenum and of exocrine pancreatic secretion of calves. The collection of the exocrine pancreatic secretion of calves from a duodenal cannula. Br. J. Nutr. 29, 387-397.

Ternouth J.H., Roy J.H.B., Stobo I.J.F., Ganderton P., Gillies C.M., Shotton S.M., 1974. The effect of experimental variation in the quantity of pancreatic secretion on the digestion and utilization of milksubtitute diets by the calf. Br. J. Nutr. 32,37-45.
Ternouth J.H., Roy J.H.B., Thompson S.Y., Toothill J., Gillies C.M., Edward-Webb J.D., 1975. Concurrent studies of the flow of digesta in the duodenum and of exocrine pancreatic secretion of calves. 3. Further studies on the addition of fat to skim and the use of non-milk proteins in milksubstitute diets. Br. J. Nutr. 33, 181-196.

Thompson J.C., Greeley G.H., Rayford P.L., Thownsend C.M., 1987. Gastrointestinal endocrinology, 447pp. Mc Graw-Hill, New-York.

Toullec R., Guilloteau P., 1989. Research into the digestive physiology of the milk-fed calf. In: Nutrition and Digestive Physiology. I. Monogastric Farm Animals (E.J. Van Weerden \& J. Huisman, eds), Pudoc, Wageningen, 37-55.

Toullec R., Chayvialle J.A., Guilloteau P., Bernard C., 1992. Early-life pattern of plasma gut regulatory peptide levels in calves. Effect of age, weaning and feeding. Comp. Biochem. Physiol. 102A, 203-209.

Zabielski R., Podgurniak P., Pierzynowski S.G., Barej W., 1990. Exocrine pancreatic function during cold blockade of the vagus in chronic experiments on calves. Exp. Physiol. 75, 401-406.

\section{Summary}

Digestive secretions and their regulation in the young preruminant calf.

The role of digestive secretions is important in transforming food into nutrients that are able to be absorbed and assimilated by the organism. The general principles of the regulation of their synthesis and release are described, underlining the complexity of the mechanisms involved. Examples are given to show the effect of some factors (meals, protein sources, age, digestibility) on gastric and pancreatic secretions and to suggest an explanation of the mechanisms involved. Some illustrations show the possible applications in nutrition or clinical field, in animals and humans. In conclusion, the results obtained using new techniques at our disposal and combining them with classical methods, allow us to open up new ways of investigation. These will probably bring to light new knowledge which can be used in the regulation of secretions in order to adapt them better to the food given, taking the rearing environment into account.

GUILLOTEAU P., LE HUËROU-LURON I., QUILLET J., TOULLEC R., 1994. Les sécrétions digestives et leur régulation chez le jeune veau préruminant. INRA Prod. Anim., 7 (2), 85-95. 